PROCEEDINGS OF THE

AMERICAN MATHEMATICAL SOCIETY

Volume 140, Number 5, May 2012, Pages 1585-1592

S 0002-9939(2012)10976-1

Article electronically published on January 4, 2012

\title{
LORENTZ NORM INEQUALITIES FOR THE HARDY OPERATOR INVOLVING SUPREMA
}

\author{
DMITRY V. PROKHOROV
}

(Communicated by Richard Rochberg)

\begin{abstract}
The weighted Lorentz norm inequalities for the Hardy operator involving suprema are characterized.
\end{abstract}

\section{INTRODUCTION}

Let $b \in(0, \infty]$. Denote by $\mathfrak{M}^{+}$the class of all nonnegative Lebesgue measurable functions on $(0, b)$. If $w, f \in \mathfrak{M}^{+}$, the distribution function $f_{*}$ of $f$ with respect to $w$ is given by

$$
f_{*}(x)=\int_{\{|f|>x\}} w, x \in[0, \infty) .
$$

For $0<p<\infty, 0<q \leq \infty$ and $w \in \mathfrak{M}^{+}$, the Lorentz space $L^{p, q}(w)$ consists of all functions $f$ satisfying $\|f\|_{L^{p, q}(w)}<\infty$, where

$$
\|f\|_{L^{p, q}(w)}= \begin{cases}{\left[q \int_{0}^{\infty} t^{q-1} f_{*}(t)^{\frac{q}{p}} d t\right]^{\frac{1}{q}}} & \text { for } q \in(0, \infty), \\ \sup _{t \in(0, \infty)} t f_{*}(t)^{\frac{1}{p}} & \text { for } q=\infty .\end{cases}
$$

In case $p=q$ the Lorentz space $L^{p, q}(w)$ coincides with the Lebesgue space $L^{q}(w)$ with the norm $\|f\|_{L^{q}(w)}=\left\|f w^{\frac{1}{q}}\right\|_{q}$.

The main use of the Lorentz space is the interpolation theory, where the standard reference is [2], as well as many other applications. However, the study of the mapping properties of operators of classical analysis in the Lorentz spaces had also attracted much attention. In particular, it is concerned with the weighted Hardy type operators, for which boundedness in the Lorentz spaces was intensively studied by many authors. We mention here just a few of the contributors $1,13,4,5,9,11$, 12.

On the other hand, some operators involving suprema had been recently encountered in various research projects; see [6, 7] for the weighted Hardy operators, and [10] with the supplement for the Riemann-Liouville fractional integrals. These operators may be found in many branches of analysis and applications to PDE and function spaces theory (see [6] for additional references).

Received by the editors August 12, 2010 and, in revised form, November 1, 2010.

2010 Mathematics Subject Classification. Primary 26D15; Secondary 47G10.

Key words and phrases. Weighted Lorentz norm inequalities, Hardy operator involving suprema.

(C)2012 American Mathematical Society Reverts to public domain 28 years from publication 1585 
In detail the paper [6] deals with the characterization of the weighted Lebesgue norm inequalities for the operator

$$
(T f)(x)=\sup _{x \leq \tau<b} u(\tau) \int_{0}^{\tau} f(y) \sigma(y) d y,
$$

where $u$ is a continuous function on $(0, b), \sigma \in \mathfrak{M}^{+}$.

We characterize weighted Lorentz norm inequalities

$$
\|T f\|_{L^{p, q}(w)} \leq C\|f\|_{L^{r, s}(v)}\left(f \in \mathfrak{M}^{+}\right)
$$

for the Hardy operator involving suprema (0.1).

Throughout this paper, $A \lesssim B$ and $B \gtrsim A$ mean that $A \leq c B$, where the constant $c$ depends only on $p, q, \alpha$ and may be different in different places. If both $A \lesssim B$ and $A \gtrsim B$, then we write $A \approx B$.

\section{MAIN RESUlts}

First we will give a couple of auxiliary results.

Lemma 1.1. Let $g$ be a continuous function on $(0, b)$ such that

$$
f(x):=\sup _{\tau \in[x, b)} g(\tau)<\infty
$$

for all $x \in(0, b)$. Then $f$ is a continuous on $(0, b)$.

In paper [11] it was shown (see [11, equality (2.6)]) that

$$
\|h\|_{L^{p, q}(w)}=\left(\frac{q}{p}\right)^{\frac{1}{q}}\|h\|_{L^{q}(\tilde{w})},
$$

where $\tilde{w}(x)=w(x)\left(\int_{x}^{\infty} w\right)^{\frac{q}{p}-1}$, for an arbitrary nonnegative nondecreasing function $h$. The following lemma is an analogue of this result for a nonnegative nonincreasing function.

Lemma 1.2. Let $h$ be a nonincreasing and nonnegative function on $(0, b), w \in \mathfrak{M}^{+}$, $p, q \in(0, \infty)$, and $\rho(x)=w(x)\left(\int_{0}^{x} w\right)^{\frac{q}{p}-1}$. Then

$$
\|h\|_{L^{p, q}(w)}=\left(\frac{q}{p}\right)^{\frac{1}{q}}\|h\|_{L^{q}(\rho)} .
$$

The main results are contained in the following two theorems.

Theorem 1.3. Let $r, q \in(1, \infty), s \in[1, \infty], p \in(0, \infty), \max \{r, s\} \leq q$. Let $u$ be a continuous function on $(0, b), w, v, \sigma \in \mathfrak{M}^{+}, \tilde{u}(x)=\sup _{x \leq \tau<b} u(\tau)$. The inequality (0.2) holds iff $A<\infty$, where

$$
A:=\sup _{0<x<b}\left[\tilde{u}(x)\left[\int_{0}^{x} w\right]^{\frac{1}{p}}+\left[\int_{x}^{b} \tilde{u}(t)^{q}\left[\int_{0}^{t} w\right]^{\frac{q}{p}-1} w(t) d t\right]^{\frac{1}{q}}\right]\left\|\frac{\sigma \chi_{(0, x]}}{v}\right\|_{L^{r^{\prime}, s^{\prime}}(v)} .
$$

Proof. Sufficiency. By [6, Lemma 2.2 (i)], we have

$$
(T f)(x)=\sup _{x \leq \tau<b} \tilde{u}(\tau) \int_{0}^{\tau} f(y) \sigma(y) d y .
$$


Let $\left\{b_{n}\right\}_{1}^{\infty} \subset(0, b)$ be a sequence such that $b_{n} \uparrow b$ as $n \rightarrow \infty$. Fix an arbitrary function $f \in \mathfrak{M}^{+}$and put $f_{n}:=\min \{n, f\} \chi_{\left(0, b_{n}\right]}, \sigma_{n}:=\min \{n, \sigma\}, \tilde{u}_{n}:=\min \{n, \tilde{u}\}$. Then

$$
\left(T_{n} f_{n}\right)(x)=\sup _{x \leq \tau<b} \tilde{u}_{n}(\tau) \int_{0}^{\tau} f_{n}(y) \sigma_{n}(y) d y<\infty
$$

for all $x \in(0, b)$.

Fix an arbitrary $n \in \mathbb{N}$. We will prove the following relation:

$$
\left\|T_{n} f_{n}\right\|_{L^{p, q}(w)} \lesssim A\|f\|_{L^{r, s}(v)} .
$$

If $\left(T_{n} f_{n}\right)(x)=0$ for every $x \in(0, b)$, then (1.1) holds. So we assume that there exists a point $x_{0} \in(0, b)$ such that $\left(T_{n} f_{n}\right)\left(x_{0}\right)>0$. Put $E_{k}:=\left\{x \in(0, b) \mid\left(T_{n} f_{n}\right)(x)>\right.$ $\left.2^{k}\right\}, k \in \mathbb{Z}$, and

$$
K_{1}:=\inf \left\{k \in \mathbb{Z} \mid(0, b) \backslash E_{k} \neq \varnothing\right\}-1, K_{2}:=\max \left\{k \in \mathbb{Z} \mid E_{k} \neq \varnothing\right\}+1 .
$$

We have

$$
\begin{aligned}
& \left\|T_{n} f_{n}\right\|_{L^{p, q}(w)}^{q}=\sum_{k \in \mathbb{Z}} \int_{2^{k}}^{2^{k+1}} q t^{q-1}\left(T_{n} f_{n}\right)_{*}(t)^{\frac{q}{p}} d t \lesssim \sum_{k \in \mathbb{Z}} 2^{k q}\left(\int_{E_{k}} w\right)^{\frac{q}{p}} \\
& =\sum_{k<K_{2}} 2^{k q}\left(\int_{E_{k}} w\right)^{\frac{q}{p}} \approx 2^{K_{1} q}\left(\int_{0}^{b} w\right)^{\frac{q}{p}}+\sum_{K_{1}<k<K_{2}} 2^{k q}\left(\int_{0}^{x_{k}} w\right)^{\frac{q}{p}},
\end{aligned}
$$

where $x_{k}:=\sup E_{k}, K_{1}<k<K_{2}$. Note that $\left(T_{n} f_{n}\right)\left(x_{k}\right)=2^{k}$ for $K_{1}<k<K_{2}$ by the continuity (by Lemma 1.1) of the function $T_{n} f_{n}$. Since $\left(T_{n} f_{n}\right)\left(x_{k-1}\right)=2^{k-1}<$ $\left(T_{n} f_{n}\right)\left(x_{k}\right)$, we have that

$$
\left(T_{n} f_{n}\right)\left(x_{k}\right)=\sup _{x_{k} \leq \tau \leq x_{k-1}} \tilde{u}_{n}(\tau) \int_{0}^{\tau} f_{n} \sigma_{n}>u_{n}\left(x_{k-1}\right) \int_{0}^{x_{k-1}} f_{n} \sigma_{n} .
$$

The first term of (1.2) is estimated in the following way:

$$
\begin{aligned}
& 2^{K_{1} q}\left(\int_{0}^{b} w\right)^{\frac{q}{p}}=\sup _{x>b_{n}} 2^{K_{1} q}\left(\int_{0}^{x} w\right)^{\frac{q}{p}} \lesssim \sup _{x>b_{n}}\left(T_{n} f_{n}\right)(x)^{q}\left(\int_{0}^{x} w\right)^{\frac{q}{p}} \\
& =\sup _{x>b_{n}} \tilde{u}_{n}(x)^{q}\left(\int_{0}^{x} w\right)^{\frac{q}{p}}\left[\int_{0}^{x} f_{n} \sigma_{n}\right]^{q} \\
& \leq \sup _{x>b_{n}} \tilde{u}_{n}(x)^{q}\left(\int_{0}^{x} w\right)^{\frac{q}{p}}\left\|f_{n}\right\|_{L^{r, s}(v)}^{q}\left\|\frac{\sigma_{n} \chi_{(0, x]}}{v}\right\|_{L^{r^{\prime}, s^{\prime}}(v)}^{q} \leq A^{q}\|f\|_{L^{r, s}(v)}^{q} .
\end{aligned}
$$

For the estimate of the second term of (1.2) we put $x_{K_{1}}=b, x_{K_{2}}=0$ and

$$
W_{k}:= \begin{cases}\int_{x_{k+1}}^{x_{k}} w(t)\left(\int_{0}^{t} w\right)^{\frac{q}{p}-1} d t, & q<p, \\ \left(\int_{x_{k+1}}^{x_{k}} w\right)^{\frac{q}{p}}, & q \geq p .\end{cases}
$$

Observe that

$$
W_{k} \lesssim \min \left\{\int_{x_{k+1}}^{x_{k}} w(t)\left(\int_{0}^{t} w\right)^{\frac{q}{p}-1} d t,\left(\int_{x_{k+1}}^{x_{k}} w\right)^{\frac{q}{p}}\right\} .
$$


In the case of $q<p$ we obtain

$$
\begin{aligned}
\sum_{K_{1}<k<K_{2}} 2^{k q} & \left(\int_{0}^{x_{k}} w\right)^{\frac{q}{p}}=\frac{q}{p} \sum_{K_{1}<k<K_{2}} 2^{k q} \int_{0}^{x_{k}} w(t)\left(\int_{0}^{t} w\right)^{\frac{q}{p}-1} d t \\
= & \frac{q}{p} \sum_{K_{1}<k<K_{2}} 2^{k q} \sum_{k \leq m<K_{2}} \int_{x_{m+1}}^{x_{m}} w(t)\left(\int_{0}^{t} w\right)^{\frac{q}{p}-1} d t \\
& =\frac{q}{p} \sum_{K_{1}<m<K_{2}} W_{m} \sum_{K_{1}<k \leq m} 2^{k q} \approx \sum_{K_{1}<m<K_{2}} 2^{m q} W_{m} .
\end{aligned}
$$

If $q \geq p$, then by [8, Proposition 2.1], we estimate

$$
\begin{aligned}
\sum_{K_{1}<k<K_{2}} 2^{k q}\left(\int_{0}^{x_{k}} w\right)^{\frac{q}{p}}= & \sum_{K_{1}<k<K_{2}} 2^{k q}\left(\sum_{k \leq m<K_{2}} \int_{x_{m+1}}^{x_{m}} w\right)^{\frac{q}{p}} \\
& \approx \sum_{K_{1}<k<K_{2}} 2^{k q}\left(\int_{x_{k+1}}^{x_{k}} w\right)^{\frac{q}{p}}=\sum_{K_{1}<k<K_{2}} 2^{k q} W_{k} .
\end{aligned}
$$

In both cases, we have

$$
\begin{aligned}
\sum_{K_{1}<k<K_{2}} 2^{k q}\left(\int_{0}^{x_{k}} w\right)^{\frac{q}{p}} \approx & \sum_{K_{1}<k<K_{2}}\left(T_{n} f_{n}\right)\left(x_{k}\right)^{q} W_{k} \\
= & \sum_{K_{1}<k<K_{2}}\left(\sup _{x_{k} \leq \tau \leq x_{k-1}} \tilde{u}_{n}(\tau) \int_{0}^{\tau} f_{n} \sigma_{n}\right)^{q} W_{k} \\
& =\sum_{K_{1}<k<K_{2}}\left(\tilde{u}_{n}\left(z_{k}\right) \int_{0}^{z_{k}} f_{n} \sigma_{n}\right)^{q} W_{k}=S_{1}+S_{2},
\end{aligned}
$$

where $z_{k} \in\left[x_{k}, x_{k-1}\right), K_{1}<k<K_{2}$, such that (see (1.3))

$$
\begin{gathered}
\left(T_{n} f_{n}\right)\left(x_{k}\right)=\sup _{x_{k} \leq \tau \leq x_{k-1}} \tilde{u}_{n}(\tau) \int_{0}^{\tau} f_{n} \sigma_{n}=\tilde{u}_{n}\left(z_{k}\right) \int_{0}^{z_{k}} f_{n} \sigma_{n}, \\
S_{1}=\sum_{K_{1}<k<K_{2}}\left(\tilde{u}_{n}\left(z_{k}\right) \int_{z_{k+2}}^{z_{k}} f_{n} \sigma_{n}\right)^{q} W_{k}, z_{K_{2}}=z_{K_{2}+1}=0, \\
S_{2}=\sum_{K_{1}<k<K_{2}-2}\left(\tilde{u}_{n}\left(z_{k}\right) \int_{0}^{z_{k+2}} f_{n} \sigma_{n}\right)^{q} W_{k} .
\end{gathered}
$$

Now, by (1.5), we find

$$
\begin{array}{r}
S_{1} \lesssim \sum_{K_{1}<k<K_{2}} \tilde{u}_{n}\left(z_{k}\right)^{q}\left\|f_{n} \chi_{\left[z_{k+2}, z_{k}\right]}\right\|_{L^{r, s}(v)}^{q}\left\|\frac{\sigma_{n} \chi_{\left[z_{k+2}, z_{k}\right]}}{v}\right\|_{L^{r^{\prime}, s^{\prime}}(v)}^{q}\left(\int_{0}^{z_{k}} w\right)^{\frac{q}{p}} \\
\leq A^{q} \sum_{K_{1}<k<K_{2}}\left\|f_{n} \chi_{\left[z_{k+2}, z_{k}\right]}\right\|_{L^{r, s}(v)}^{q} \lesssim A^{q}\|f\|_{L^{r, s}(v)}^{q} .
\end{array}
$$


Using the relation (1.5) and the criteria of boundedness of the Hardy operator between Lorentz spaces [5, Theorem 3] we get

$$
\begin{aligned}
& S_{2} \lesssim \sum_{K_{1}<k<K_{2}-2} \int_{x_{k+1}}^{x_{k}} w(t)\left(\int_{0}^{t} w\right)^{\frac{q}{p}-1}\left(\tilde{u}(t) \int_{0}^{t} f_{n} \sigma\right)^{q} d t \\
& \lesssim \sup _{0<x<b}\left[\int_{x}^{b} \tilde{u}(t)^{q}\left[\int_{0}^{t} w\right]^{\frac{q}{p}-1} w(t) d t\right]\left\|\frac{\sigma \chi_{(0, x]}}{v}\right\|_{L^{r^{\prime}, s^{\prime}(v)}}^{q}\|f\|_{L^{r, s}(v)}^{q} \leq A^{q}\|f\|_{L^{r, s}(v)}^{q} .
\end{aligned}
$$

Thus, (1.1) is proved.

Now we prove that for arbitrary $t \in(0, b)$,

$$
\{x \in(0, b) \mid(T f)(x)>t\}=\bigcup_{n \in \mathbb{N}}\left\{x \in(0, b) \mid\left(T_{n} f_{n}\right)(x)>t\right\} .
$$

Since $(T f)(x) \geq\left(T_{n} f_{n}\right)(x)$, the left part of (1.6) contains the right part of (1.6).

For the proof of the inverse embedding we fix a point $x \in(0, b)$ such that $(T f)(x)>t$. By the definition of $(T f)(x)$ there exists a point $z \in[x, b)$ such that $\tilde{u}(z) \int_{0}^{z} f \sigma>t$. Since

$$
\tilde{u}_{n}(z) \int_{0}^{z} f_{n} \sigma_{n} \uparrow \tilde{u}(z) \int_{0}^{z} f \sigma
$$

there exists $n_{0} \in \mathbb{N}$ such that $\tilde{u}_{n_{0}}(z) \int_{0}^{z} f_{n_{0}} \sigma_{n_{0}}>t$. Hence,

$$
\left(T_{n_{0}} f_{n_{0}}\right)(x) \geq\left(T_{n_{0}} f_{n_{0}}\right)(z) \geq \tilde{u}_{n_{0}}(z) \int_{0}^{z} f_{n_{0}} \sigma_{n_{0}}>t,
$$

and the equality (1.6) is proved.

Using (1.1) and (1.6), the Monotone Convergence Theorem implies (0.2) with the constant $C \lesssim A$.

Necessity. For arbitrary $x \in(0, b)$ we have

$$
(T f)(x) \geq \int_{0}^{x} f \sigma \cdot \sup _{x \leq \tau<b} u(\tau)=\tilde{u}(x) \int_{0}^{x} f \sigma .
$$

Put $\lambda:=(T f)(x)$. Then $(0, x] \subset\{y \in(0, b) \mid(T f)(y)>t\}$ for all $t \in(0, \lambda)$, and

$$
\begin{aligned}
C\|f\|_{L^{r, s}(v)} \geq\|T f\|_{L^{p, q}(w)} \geq & \left(\int_{0}^{\lambda} q t^{q-1}(T f)_{*}(t)^{\frac{q}{p}} d t\right)^{\frac{1}{q}} \\
& \geq \lambda\left(\int_{0}^{x} w\right)^{\frac{1}{p}} \geq\left(\int_{0}^{x} w\right)^{\frac{1}{p}} \tilde{u}(x) \int_{0}^{x} f \cdot \frac{\sigma}{v} \cdot v .
\end{aligned}
$$

Hence,

$$
C \geq\left(\int_{0}^{x} w\right)^{\frac{1}{p}} \tilde{u}(x) \sup _{f \in \mathfrak{M}^{+}, f \neq 0} \frac{\int_{0}^{x} f \cdot \frac{\sigma}{v} \cdot v}{\|f\|_{L^{r, s}(v)}} \gtrsim\left(\int_{0}^{x} w\right)^{\frac{1}{p}} \tilde{u}(x)\left\|\frac{\sigma \chi_{(0, x]}}{v}\right\|_{L^{r^{\prime}, s^{\prime}}(v)} .
$$


By Lemma 1.2 we have

$$
\begin{aligned}
\|T f\|_{L^{p, q}(w)}=\left(\frac{q}{p}\right)^{\frac{1}{q}}\|T f\|_{L^{q}(\rho)} & \geq\left[\int_{0}^{b} \frac{q}{p}\left[\int_{0}^{y} w\right]^{\frac{q}{p}-1}\left[\tilde{u}(y) \int_{0}^{y} f \sigma\right]^{q} w(y) d y\right]^{\frac{1}{q}} \\
& \geq\left(\int_{x}^{b} \frac{q}{p} \tilde{u}(y)^{q}\left[\int_{0}^{y} w\right]^{\frac{q}{p}-1} w(y) d y\right)^{\frac{1}{q}} \int_{0}^{x} f \sigma
\end{aligned}
$$

whence the estimate

$$
C \geq\left(\frac{q}{p}\right)^{\frac{1}{q}}\left(\int_{x}^{b} \tilde{u}(y)^{q}\left[\int_{0}^{y} w\right]^{\frac{q}{p}-1} w(y) d y\right)^{\frac{1}{q}}\left\|\frac{\sigma \chi(0, x]}{v}\right\|_{L^{r^{\prime}, s^{\prime}}(v)}
$$

follows.

Theorem 1.4. Let $r \in(1, \infty), s \in[1, \infty], p \in(0, \infty), q=\infty$. Let $u$ be a continuous function on $(0, b), w, v, \sigma \in \mathfrak{M}^{+}$. The inequality (0.2) holds iff $B<\infty$, where

$$
B:=\sup _{0<x<b} \tilde{u}(x)\left[\int_{0}^{x} w\right]^{\frac{1}{p}}\left\|\frac{\sigma \chi(0, x]}{v}\right\|_{L^{r^{\prime}, s^{\prime}}(v)},
$$

$\tilde{u}(x)=\sup _{x \leq \tau<b} u(\tau)$.

Proof. Sufficiency. Using the same ideas and notation as in the proof of the sufficient part of Theorem 1.3, we obtain (in case $q=\infty$ ) the relation

$$
\left\|T_{n} f_{n}\right\|_{L^{p, \infty}(w)} \lesssim \max \left\{2^{K_{1}}\left(\int_{0}^{b} w\right)^{\frac{1}{p}} ; \sup _{K_{1}<k<K_{2}} 2^{k}\left(\int_{0}^{x_{k}} w\right)^{\frac{1}{p}}\right\},
$$

instead of (1.2). In the same way as (1.4) we prove the bound

$$
2^{K_{1}}\left(\int_{0}^{b} w\right)^{\frac{1}{p}} \lesssim B\|f\|_{L^{r, s}(v)} .
$$

For estimation of the second term of the right part of (1.7), we write

$$
\begin{aligned}
\sup _{K_{1}<k<K_{2}} 2^{k}\left(\int_{0}^{x_{k}} w\right)^{\frac{1}{p}} \approx \sup _{K_{1}<k<K_{2}}\left(T_{n} f_{n}\right)\left(x_{k}\right)\left(\int_{0}^{x_{k}} w\right)^{\frac{1}{p}} & \\
& =\sup _{K_{1}<k<K_{2}} \tilde{u}_{n}\left(z_{k}\right) \int_{0}^{z_{k}} f_{n} \sigma_{n}\left(\int_{0}^{x_{k}} w\right)^{\frac{1}{p}}=S_{3},
\end{aligned}
$$

where $z_{k} \in\left[x_{k}, x_{k-1}\right), K_{1}<k<K_{2}$, such that

$$
\left(T_{n} f_{n}\right)\left(x_{k}\right)=\sup _{x_{k} \leq \tau \leq x_{k-1}} \tilde{u}_{n}(\tau) \int_{0}^{\tau} f_{n} \sigma_{n}=\tilde{u}_{n}\left(z_{k}\right) \int_{0}^{z_{k}} f_{n} \sigma_{n} .
$$

Now, we find

$$
\begin{aligned}
S_{3} & \lesssim \sup _{K_{1}<k<K_{2}} \tilde{u}_{n}\left(z_{k}\right)\left\|f_{n} \chi_{\left(0, z_{k}\right]}\right\|_{L^{r, s}(v)}\left\|\frac{\sigma_{n} \chi_{\left(0, z_{k}\right]}}{v}\right\|_{L^{r^{\prime}, s^{\prime}(v)}}\left(\int_{0}^{z_{k}} w\right)^{\frac{1}{p}} \\
& \leq B\|f\|_{L^{r, s}(v)} .
\end{aligned}
$$


Thus, (1.1) with $q=\infty$ is proved and the Monotone Convergence Theorem implies (0.2) with $C \lesssim B$.

Necessity. For arbitrary $x \in(0, b)$ we put $\lambda:=(T f)(x)$. Then $(0, x] \subset\{y \in$ $(0, b) \mid(T f)(y)>t\}$ for all $t \in(0, \lambda)$, and

$$
\begin{aligned}
C\|f\|_{L^{r, s}(v)} & \geq\|T f\|_{L^{p, \infty}(w)} \\
& \geq \sup _{t \in(0, \lambda)} t(T f)_{*}(t)^{\frac{1}{p}} \geq \lambda\left(\int_{0}^{x} w\right)^{\frac{1}{p}} \geq\left(\int_{0}^{x} w\right)^{\frac{1}{p}} \tilde{u}(x) \int_{0}^{x} f \cdot \frac{\sigma}{v} \cdot v .
\end{aligned}
$$

This implies $C \gtrsim B$.

By using results of the paper [6] and Lemma 1.2 it is possible to characterize the inequality (0.2) in the case of $r=s, q<\infty$. We note here a case which is not contained in Theorem 1.3 and follows from [6. Theorem 4.4] and Lemma 1.2 .

Theorem 1.5. Let $s=r, p \in(0, \infty), 1<q<r<\infty, \frac{1}{\nu}=\frac{1}{q}-\frac{1}{r}$. Let $u$ be a continuous function on $(0, b), \sigma \equiv 1, w, v \in \mathfrak{M}^{+}, \rho(x)=w(x)\left(\int_{0}^{x} w\right)^{\frac{q}{p}-1}$, $\tilde{u}(x)=\sup _{x \leq \tau<b} u(\tau)$. The inequality (0.2) holds iff

$$
\int_{0}^{b}\left(\int_{t}^{b} \tilde{u}(s)^{q} \rho(s) d s\right)^{\frac{\nu}{r}} \tilde{u}(t)^{q}\left(\int_{0}^{t} v^{1-r^{\prime}}\right)^{\frac{\nu}{r^{\prime}}} \rho(t) d t<\infty
$$

and

$$
\int_{0}^{b}\left(\int_{t}^{b} \rho\right)^{\frac{\nu}{r}} \sup _{t \leq \tau<b}\left[\tilde{u}(\tau)^{\nu}\left(\int_{0}^{\tau} v^{1-r^{\prime}}\right)^{\frac{\nu}{r^{\prime}}}\right] \rho(t) d t<\infty .
$$

\section{REFERENCES}

[1] K. F. Andersen and B. Muckenhoupt. Weighted weak type Hardy inequalities with applications to Hilbert transforms and maximal functions. Stud. Math., 72:9-26, 1982. MR665888 $(83 \mathrm{k}: 42018)$

[2] C. Bennett and R. Sharpley. Interpolation of Operators. Pure and Applied Mathematics 129, Academic Press, Inc., Boston, MA, 1988. MR928802 (89e:46001)

[3] M. J. Carro and J. Soria. Weighted Lorentz spaces and the Hardy operator. J. Funct. Anal., 112(2):480-494, 1993. MR.1213148 (94f:42025)

[4] H.-M. Chung, R. A. Hunt, and D. S. Kurtz. The Hardy-Littlewood maximal function on $L(p, q)$ spaces with weights. Indiana Univ. Math. J., 31:109-120, 1982. MR642621 (83b:42021)

[5] D. E. Edmunds, P. Gurka, and L. Pick. Compactness of Hardy-type integral operators in weighted Banach function spaces. Stud. Math., 109(1):73-90, 1994. MR.1267713 (95c:47033)

[6] A. Gogatishvili, B. Opic, and L. Pick. Weighted inequalities for Hardy-type operators involving suprema. Collect. Math., 57(3):227-255, 2006. MR2264321 (2007g:26019)

[7] A. Gogatishvili and L. Pick. A reduction theorem for supremum operators. J. Comput. Appl. Math., 208(1):270-279, 2007. MR2347749 (2009a:26013)

[8] M. Gol'dman, H. Heinig, and V. Stepanov. On the principle of duality in Lorentz spaces. Can. J. Math., 48(5):959-979, 1996. MR1414066 (97h:42008)

[9] E. Lomakina and V. Stepanov. On the compactness and approximation numbers of Hardytype integral operators in Lorentz spaces. J. Lond. Math. Soc., II. Ser., 53(2):369-382, 1996. MR.1373067 (97f:47031)

[10] D. V. Prokhorov. Inequalities for Riemann-Liouville operator involving suprema. Collect. Math., 61(3):263-276, 2010. MR2732371(2011j:26026) 
[11] E. Sawyer. Weighted Lebesgue and Lorentz norm inequalities for the Hardy operator. Trans. Amer. Math. Soc., 281:329-337, 1984. MR719673 (85f:26013)

[12] V. D. Stepanov. Weighted norm inequalities for integral operators and related topics. Krbec, Miroslav (ed.) et al., Nonlinear analysis, function spaces and applications. Vol. 5. Proceedings of the spring school held in Prague, May 23-28, 1994. Prague: Prometheus Publishing House. 139-175, 1994. MR1322312 (96m:26019)

Computing Centre of the Far Eastern Branch of the Russian Academy of Sciences, Kim Yu Chen 65, Khabarovsk 680000, Russia

E-mail address: prohorov@as.khb.ru 